Research Paper

\title{
Ecdysone oxidase and 3-dehydroecdysone-3 $\beta$ - reductase contribute to the synthesis of ecdysone during early embryonic development of the silkworm
}

\author{
Cheng-Fang Wang, Ze Zhang ${ }^{\bowtie}$, Wei Sun ${ }^{\bowtie}$ \\ Laboratory of Evolutionary and Functional Genomics, School of Life Sciences, Chongqing University, Chongqing 401331, China \\ $\triangle$ Corresponding authors: Dr. Wei Sun, E-mail: sunwei077@cqu.edu.cn and Prof. Ze Zhang, E-mail: zezhang@cqu.edu.cn; Tel: +86-23-65678449 \\ (c) Ivyspring International Publisher. This is an open access article distributed under the terms of the Creative Commons Attribution (CC BY-NC) license \\ (https://creativecommons.org/licenses/by-nc/4.0/). See http://ivyspring.com/terms for full terms and conditions.
}

Received: 2018.03.22; Accepted: 2018.07.15; Published: 2018.08.06

\begin{abstract}
Maternal ecdysteroids regulate a variety of cellular processes during early embryonic development of insects, yet little is known about the genes involved in the biosynthesis of these hormones. In this study, we found that ecdysone oxidase (EO) gene, which encodes an enzyme to catalyze ecdysone (or 20-hydroxyecdysone, 20E) to 3-dehydroecdysone (3DE), was highly expressed in the mature ovaries of the domestic silkworm, Bombyx mori. B. mori EO (BmEO) was localized in the cytoplasm around the yolk granules of oocyte. Furthermore, the down-regulated expression of the BmEO gene using RNA interference could not affect normal development of the female silkworm, but lower the $20 \mathrm{E}$ titer and hatching rate of its offspring. Rescue experiments by injecting the product (3DE) of BmEO can significantly elevate the $20 \mathrm{E}$ level and hatching rate of the BmEO RNAi offspring. Meanwhile, during embryonic stage, the down-regulating expression of 3DE-3ß-reductase, which can reduce 3DE into ecdysone, also lowered the $20 \mathrm{E}$ titer. Taken together, our results prove that $3 \mathrm{DE}$ can be synthesized from ecdysone in maternal ovary yolk granules, and then the maternal 3DE is converted into active ecdysone during the early embryonic development of offspring. Thus, our findings reveal a new pathway to explain the origin of high $20 \mathrm{E}$ level before the formation the prothoracic gland in the silkworm.
\end{abstract}

Key words: ecdysone; ecdysone oxidase; 3DE-3 $\beta$-reductase; RNA interference; silkworm

\section{Introduction}

Ecdysteroids are a class of the most important hormones in insects. They can regulate insects' molting process and metamorphosis. In general, ecdysteroids are thought to be biosynthesized in the prothoracic glands (PGs) during the larval stages [1]. However, more and more studies have shown that various forms of ecdysteroids (for example: ecdysone, 20E, 2-deoxy-ecdysone and 20E 22-phosphate) are present in eggs of different insects, such as Bombyx mori, Locusta migratoria and Drosophila melanogaster [2-8]. Furthermore, these ecdysteroids play important roles in the insect embryonic development. For example, the embryonic cuticulogenesis of locust needs high ecdysteroid titer [9]. Ecdysteroids were also required for germ band elongation of the
Drosophila embryo [10]. Moreover, ecdysteroids could control embryonic diapause in different insects [11-13]. Thus, it is very important to understand the molecular mechanism of ecdysteroids synthesis at embryonic stages.

As shown above, ecdysteroids are mainly synthesized and secreted from PGs. However, previous studies have found that the early embryonic development of insects was independently regulated by the PGs $[14,15]$. In addition, the embryonic active ecdysteroids is present before PGs formation. Most importantly, a previous study has suggested that high titers of $20 \mathrm{E}$ in $B$. mori eggs are synthesized before the formation of the embryonic prothoracic glands [7]. During the embryonic development, the main 
resources in eggs including nutrient are provided by maternal ovary. Ovarian follicle of many insects can also synthesize ecdysteroids [4, 16-18]. These hormones could take part in the development of oocytes. Besides, hormones are also converted to inactive form and stored in the oocytes of insects [19, 20]. Thus, the embryonic ecdysteroids may be maternal origination. This hypothesis was firstly proposed in 1974 by Hanaoka and Ohnishi [21]. The genes involved in this process were characterized and cloned in B. mori. Yamada and Sonobe (2003) identified an ecdysteroid-phosphate phosphatase which can covert 20-hydroxyecdysone 22-phosphate $(20 \mathrm{E} 22 \mathrm{P})$ and ecdysone 22-phosphate (E22P) into free ecdysteroids in the domestic silkworm embryo [22]. Later, they further cloned the ecdysone 22-kinase (EcK) involved in the phosphorylation at the C-22 position of ecdysteroids in the ovaries of the silkworm [23].

The ecdysone titer is crucial for normal development of the insect embryo. Thus, there should be redundancy of ecdysone synthesis in insects. In other words, insects may have several pathways to produce free ecdysteroids for their development. 3-Epimerization of ecdysteroid is one of the major metabolism pathways of the hormone in insects, especially in the Lepidopteran species [24, 25]. Ecdysone (or 20E) is firstly converted to 3-dehydroecdysone (3DE or 3D20E) by ecdysone oxidase (EO), and then 3DE-3a-reductase catalyzed 3DE to 3-epiecdysone. Similar to phosphorylation of ecdysteroids shown above, the intermediate product, $3 \mathrm{DE}$ (or 3D20E) can also be reversibly reduced to active ecdysone by 3DE-3 $\beta$-reductase [26]. Previous studies have shown that most lepidopteran insects can store a large amount of $3 \mathrm{DE}$ in their prothoracic glands at immature stages [27, 28]. When the insects need, 3DE is released and rapidly reduced to ecdysone. In other words, 3DE could serve as a substrate to synthesize ecdysone. Thus, the reversible pathway between ecdysone and 3DE implies that it may take part in the synthesis of embryonic active ecdysteroids. In this study, we have characterized the function of the silkworm (B. mori) EO (BmEO) and $3 D E-3 \beta$-reductase genes and demonstrated how the silkworm utilizes this pathway to regulate the ecdysone titer of the embryo.

\section{Materials and Methods}

\section{Silkworm strain}

The polyvoltine strain N4 of the silkworm ( $B$. mori, a non-diapause strain) was fed with fresh mulberry leaves and reared at $25^{\circ} \mathrm{C}$ under a $12 / 12-\mathrm{h}$ light/dark photoperiod. For the N4 strain silkworm, newly laid egg needs 9 days to hatch, and the pupa needs 9-10 days for eclosion.

\section{Sample preparation}

For pupa, different tissues including head, fat body, hemocytes and testis/ovary were dissected from Day 7 of the male and female bodies, respectively. To determine the number of choriogenic follicles, the ovarioles were dissected from Day 7 of the female pupae and transferred to $90 \%$ glycerol and incubated at room temperature. The first choriogenic follicle on the ovariole string was identified as previously described [29]. In addition, ovaries were also dissected from female pupae at different pupal development time points. All the tissues shown above were dissected from 3-5 individuals and immediately frozen in liquid nitrogen, respectively.

For embryonic stage, the eggs from N4 strain were collected from Day 1 to Day 8 after egg-laying. For each stage, 30 eggs were collected.

\section{RNA interference}

Based on the cDNA sequences of the silkworm (B. mori) EO (BmEO, GenBank accession number: JF433972) and 3DE-3 $\beta$-reductase genes (GenBank accession number: KU233524), we designed specific primers containing the T7 promoter sequence. The primers were listed in Table S1. The PCR products were sequenced to confirm the specificity of the primers. The amplified fragments were then used as templates to generate double-strand RNAs (dsRNAs). The dsRNAs were synthesized in vitro by Ribo-MAX Large Scale RNA Production systems-T7 (Promega) using a manual method. Concentrations of the dsRNAs were quantified by UV spectrum absorbance. According to our and other previous studies, $10 \mu \mathrm{l}$ aqueous solution containing $30 \mu \mathrm{g} B m E O$ dsRNAs were injected into the Day 5 pupa $[30,31]$. After the first injection, the same amount of dsRNAs were injected every two days until adult to ensure the effective RNAi. Then, the RNAi treated female moths were mated with untreated male moths, and their offspring were collected. In addition, about $5 \mathrm{nl} 3 \mathrm{DE}$ at a concentration of $1 \mu \mathrm{g} / \mu \mathrm{l}$ was injected into eggs laid by BmEO RNAi female adult. Twenty percent of ethanol solution was used as the control. For the $3 D E-3 \beta$-reductase gene, the newly laid eggs were used to perform the RNAi experiment. Firstly, the eggs were transferred to glass slides in the same direction. About $5 \mathrm{nl}$ dsRNA at a concentration of $500 \mathrm{ng} / \mu \mathrm{l}$ was injected into one embryo using a microinjection system (Eppendorf, Hamburg, Germany). Meanwhile, $5 \mathrm{nl} B m 3 D E-3 \beta$-reductase dsRNA at a concentration of $300 \mathrm{ng} / \mu \mathrm{l}$ and $20 \mathrm{E}$ at a concentration of $300 \mathrm{ng} / \mu \mathrm{l}$ were co-injected into newly laid eggs to perform 
rescue experiments. The injected embryos were incubated in a moist plastic box at $27^{\circ} \mathrm{C}$ until hatching. In order to examine the fluctuation of 3DE in eggs after RNAi, 3000 eggs were dissected from RNAi treated virgin female moths, and grinded in liquid nitrogen to powders. The powders were homogenized with 50 milliliter $(\mathrm{ml})$ methanol to extract polar and apolar ecdysteroids. The homogenates were sonicated and centrifuged. The pellets were re-extracted twice after centrifugation. Supernatants were collected and dried at $50{ }^{\circ} \mathrm{C}$. Dried samples were re-dissolved in $2 \mathrm{ml}$ of methanol. The extraction contained free and conjugated ecdysteroid. In our lab, we only have standard sample of some free ecdysteroid (3DE, E and 20E). Thus, we further hydrolyzed the conjugated ecdysteroids to produce their free forms using Helix hydrolases (a crude enzyme preparation from Helix pomatia, Solarbio, China). Helix crude enzymes were widely used to remove conjugates (such as phosphate and ester) of ecdysteroid, but cannot directly reduce or oxidize molting hormone [32]. After an overnight incubation at $37^{\circ} \mathrm{C}$, the samples were centrifuged, and the supernatants were used to perform high performance liquid chromatography (HPLC) analysis. HPLC analysis using a C18 No-va-Pak cartridge $(4.6 \times 250$ mm; Waters Associates) was performed on a Hitachi Primaide HPLC system (Hitachi, Japan) linked to a Dual $\lambda$ Absorbance UV detector set at $254 \mathrm{~nm}$. The mobile phase consisted of acetonitrile/0.1\% (v/v) trifluoroacetic acid in water $(22: 78, \mathrm{v} / \mathrm{v})$, and the elution rate was set at $1 \mathrm{ml} / \mathrm{min}$. The standard sample of 3DE which was kindly provided as a gift by Dr. R. Lafont (Universite Pierre et Marie Curie, Paris, France) was used to identify the product of the enzyme assay. E and 20E were purchased from Sigma.

\section{Real-time PCR}

For each sample shown above, the stored tissues were grinded in liquid nitrogen to powders. Total RNA was extracted by the Ultrapure RNA kit (Beijing CoWin Biotech, Beijing, China) and treated with DNase I (Takara Bio, Shiga, Japan) to remove the genomic DNA contamination. The RNA was quantified by the UV spectrophotometer, and then 1 $\mu \mathrm{g}$ RNA was reversely transcribed to the first strand of cDNA by the EasyScript one-step gDNA removal and cDNA synthesis SuperMix kit (TransGen Biotech). The specific primers were designed and used in the quantitative real-time PCR analysis (Table S1). The quantitative real-time PCR was performed using a real-time PCR detection system (CFX96, Bio-Rad, Hercules, CA) with a SsoAdvanced SYBR Green supermix kit (Bio-Rad). The PCR was carried out as follows: $30 \mathrm{~s}$ at $95^{\circ} \mathrm{C}$, followed by 40 cycles of 5 $\mathrm{s}$ at $95^{\circ} \mathrm{C}$ and $40 \mathrm{~s}$ at $58^{\circ} \mathrm{C}$. The B. mori ribosomal protein L3 gene was used as the reference gene.

\section{Ecdysteroid measurements}

For ecdysteroid measurements, ecdysteroids were extracted from embryos after treatment. Briefly, 100 eggs were homogenized in $1 \mathrm{ml}$ cold methanol. After centrifugation, an aliquot of supernatant was dried at $70^{\circ} \mathrm{C}$, and then the dried extract was dissolved with $150 \mu$ l enzyme immunoassay (EIA) buffer $(0.4 \mathrm{M} \mathrm{NaCl}, 1 \mathrm{mM}$ EDTA, and $0.1 \%$ BSA in 0.1 $\mathrm{M}$ phosphate buffer, $\mathrm{pH}$ : 7.0) (Sangon Biotech, China). Ecdysteroid levels were quantified via competitive enzyme-linked immunoassay kit with anti-20E rabbit antiserum (EAB25, Kerafast, Boston, MA). It should be noted that EAB25 anti-20E antibody is specific for 20E [33]. The method was based on the manufacturer's instruction.

\section{Results}

The expression patterns of the genes involved in ecdysone 3-epimerization pathway during the pupal and embryonic stages of the silkworm

Previous studies showed that ecdysone 3-epimerization pathway is mainly involved in the degradation of ecdysone during the metamorphosis stage in lepidopteran insects [26, 34, 35], although ecydsone titer also displays fluctuation during the pupal stage. Thus, in order to survey the roles of the ecdysone 3-epimerization pathway at pupal stage, we firstly examined the temporal expression patterns of the genes involved in the pathway. At the $1^{\text {st }}$ Day of the pupal stage, the expression of $B m E O$ gene was detected. The transcription level of the gene gradually increased along with the development, and reached the peak at $8^{\text {th }}$ Day after the initiation of pupal stage when the ecdysone concentration was almost undetectable (Figure 1A) [36]. This indicates that BmEO could play its role in converting ecdysone to $3 \mathrm{DE}$ during this stage. However, for the $3 D E-3 a-r e d u c t a s e$ gene involved in the second step of the 3-epimerization pathway, the expression level of the gene was rather low through the whole pupal stage (Figure 1A). Thus, we focused on the function of $B m E O$ gene during the pupal development.

We investigated the spatial expression profiles of $B m E O$ gene in different male or female silkworm tissues of the pupa at the $7^{\text {th }}$ Day. The transcription signals of the gene were detected in fat body, hemocyte, goand and weak signals in pupal head (Figure 1B). Furthermore, there is no sex bias for the expression of $B m E O$ gene in fat body, hemocyte and head (Figure 1B). However, BmEO gene was 
predominantly expressed in the female ovary, and the expression level of the gene in ovary was almost 10 times higher than that in testis $(p<0.001)$. The expression divergence of $B m E O$ gene between sexes indicates that the gene might take part in female biological process. Furthermore, we examined the expression pattern of $B m E O$ gene during the maturation of eggs (Figure 1C). BmEO gene was expressed in low level during the vitellogenic stages.
At the onset of choriogenesis $(+1 /+2)$, the expression level of $B m E O$ gene sharply increased, and reached the peak at stage $+3 /+4$. Meanwhile, we also found that the expression pattern of the silkworm ecdysone 22-kinase $(E c K)$ gene is highly consistent with that of the transcription pattern of $B m E O$ gene (Figure 1C). EcK is a known enzyme could convert ecdysone and 20E into their phosphate conjugated forms which can be accumulated in the ovary [37].

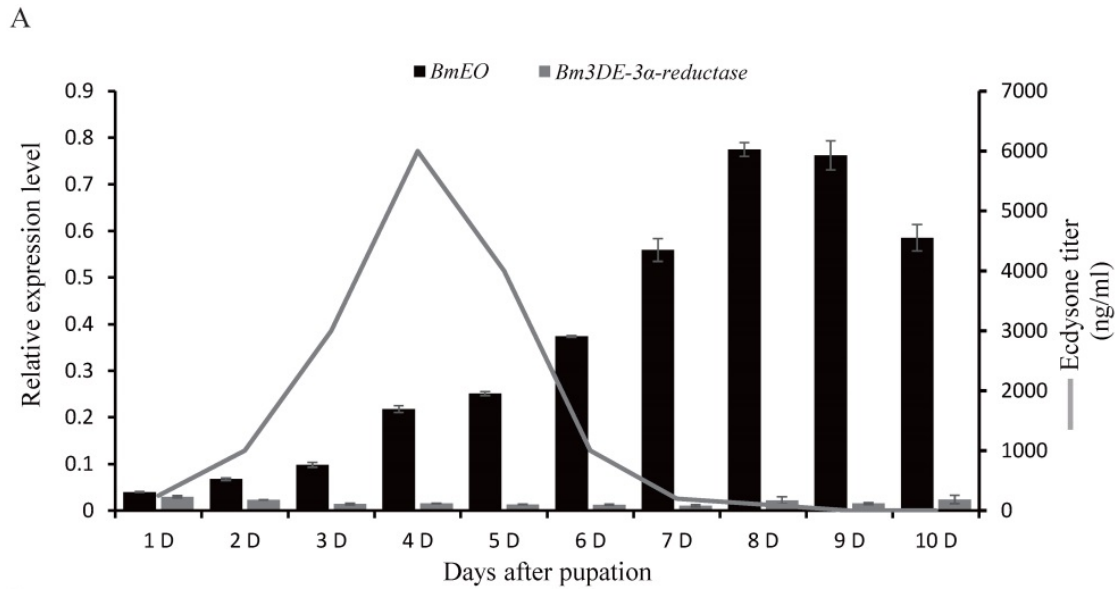

$\mathrm{B}$
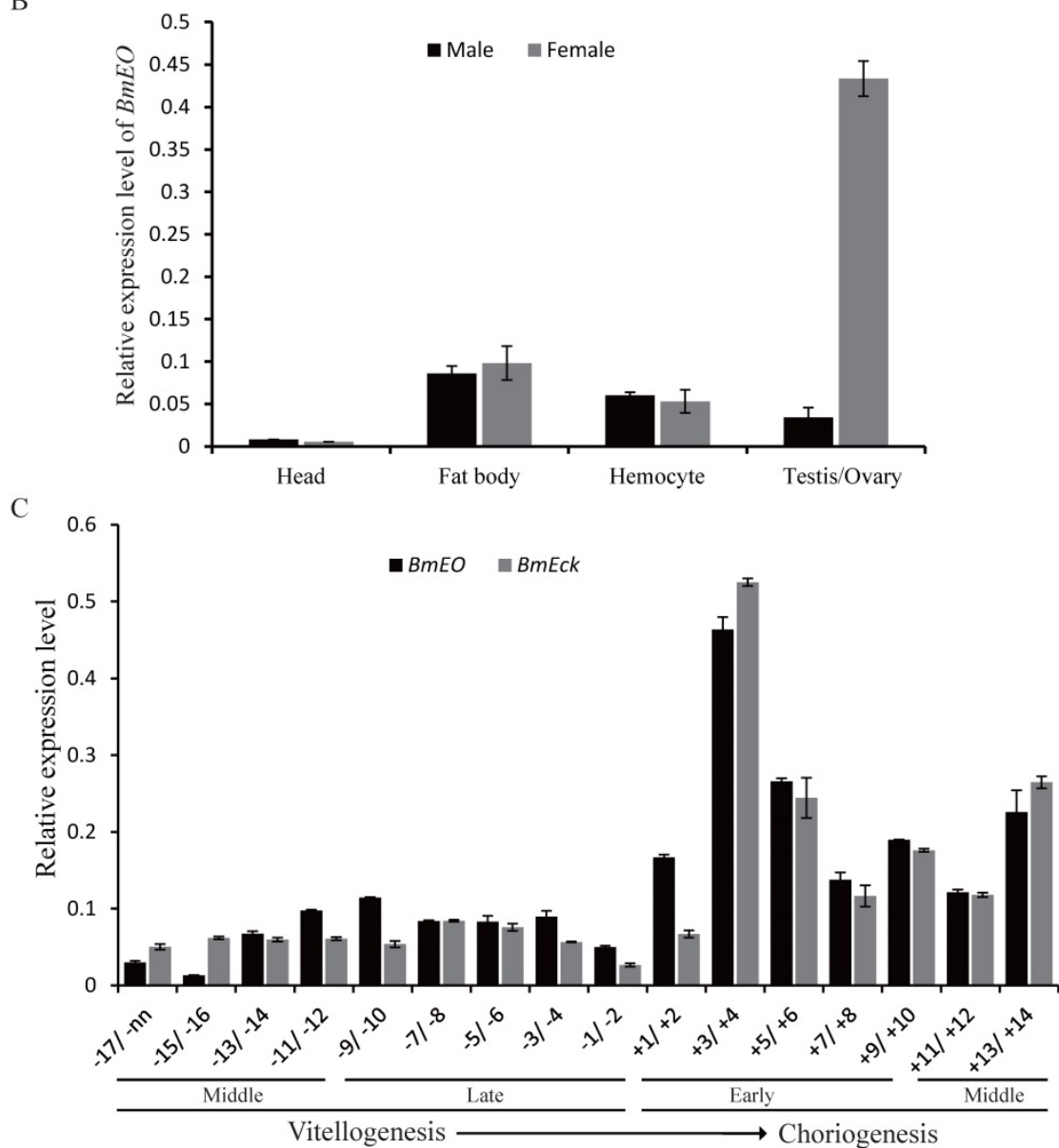

Figure 1. Expression pattern of $\mathrm{BmEO}$ and $3 D E-3 \alpha$-reductase genes during the silkworm pupal stage. (A) Temporal expression patterns of BmEO and 3DE-3a-reductase genes during the silkworm pupal stage; (B) Spatial expression pattern of BmEO gene in different male or female silkworm pupal tissues; (C) Expression pattern of $\mathrm{BmEO}$ and $\mathrm{BmEcK}$ genes in follicles at the different developmental stages. In (A), the line represents the 20E titer, modified from Kiguchi and Agui (1981). In (C), numbers indicate stages of oogenesis with respect to the start of choriogenesis $(+1$; more advanced stages of choriogenesis are numbered with increasing positive numbers, while vitellogenic stages are numbered, from the most mature one to more immature ones, with increasing negative numbers. 


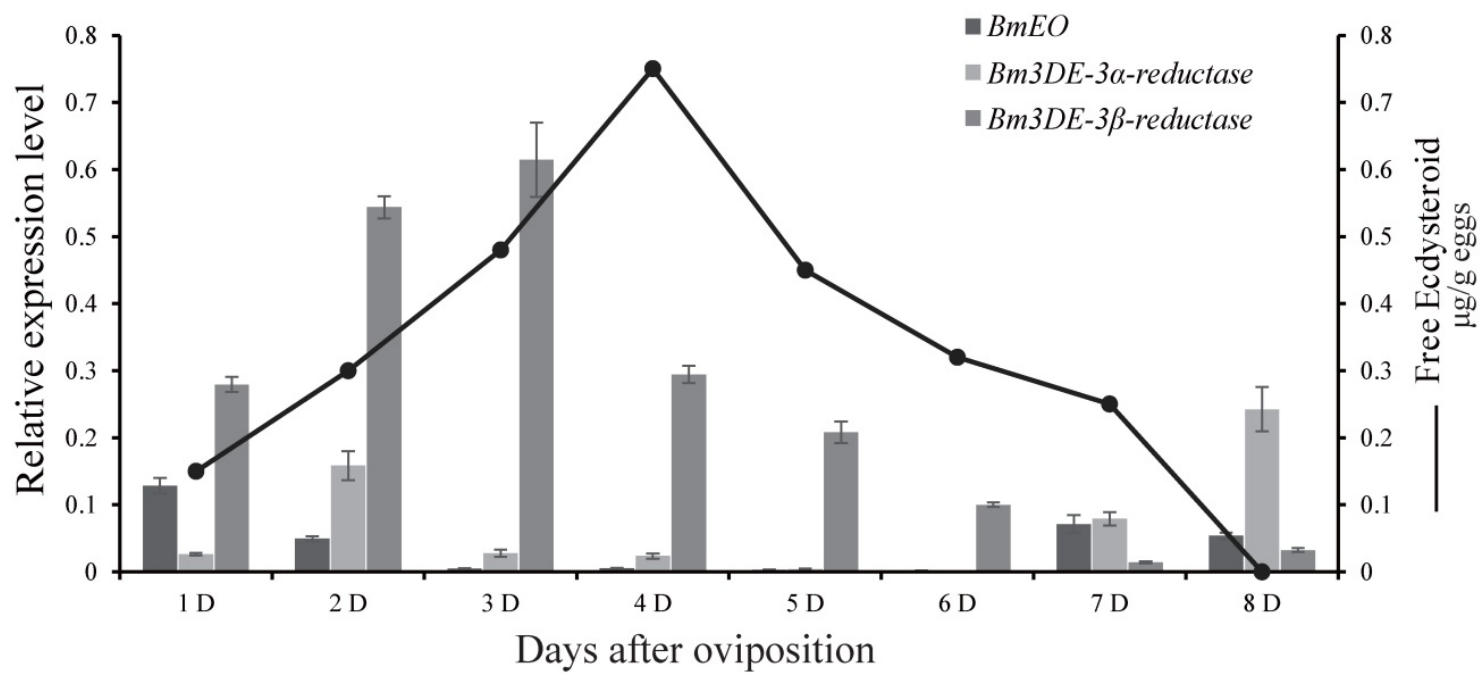

Figure 2. Expression patterns of the genes involved in ecdysone 3-epimerization pathway during the silkworm embryonic development. The line represents the 20E titer, modified from Yamada and Sonobe (2003). D represents days after egg oviposition. The data indicate means and S. E. M. of three samples after independent treatments.

At the embryonic stages, we surveyed the expression profiles of the genes involved in ecdysone 3 -epimerization pathway. Overall, the $3 D E-3 \beta$ reductase gene had much higher expression level than other two genes (EO and 3DE-3a-reductase), and it was expressed from $1^{\text {st }}$ Day after oviposition and reached a peak at $3^{\text {rd }}$ Day after ovipoision (Figure 2). Then, the expression level decreased gradually to the basal level as the embryonic development continued. Interestingly, the gene is just expressed ahead of the increase of ecdysone titer [38].

\section{The function of BmEO gene during the pupal stage of the silkworm}

As shown above, BmEO gene was prominently expressed in the pupal ovary. In order to investigate the function of the gene at this stage, we performed RNAi experiments by injecting specific dsRNA for several times. After successfully knocking-down mRNAs levels of BmEO gene (Day 7 of pupa), we examined the phenotypic variation between control and RNAi pupa (Figure 3A). We found that the BmEO-dsRNA injected silkworms grew and developed normally like EGFP-dsRNA injected silkworms. Furthermore, the eggs from control and RNAi pupa ovary were dissected, respectively. Similarly, down-regulated $B m E O$ gene expression could not affect the development of the silkworm ovary (Figure 3B). EO can convert ecdysone into 3DE. Therefore, we also examined the fluctuation of 3DE in the ovary of the female silkworm after RNAi experiment. The polar and apolar ecdysteroid were extracted from mature eggs, and hydrolyzed by Helix hydrolases. The treated ecdysteroid was further analyzed by RP-HPLC. Compared with the ovary from EGFP-dsRNA injected silkworms, the concentration of the 3DE extracted from RNAi silkworm was significantly reduced $(70.56 \pm 8.35$ vs. $49.30 \pm 6.64 \mathrm{ng} / \mu \mathrm{l}, P=0.026, \mathrm{t}$ test $)$, indicating $\mathrm{EO}$ could take part in the accumulation of 3DE in the silkworm eggs (Figure 3C, inner figure C1). However, it can be found from Figure $3 \mathrm{C}$ that the titer of $\mathrm{E}$ did not change between EGEP-dsRNA and BmEOdsRNA injected pupae. One possible reason is that 3DE converted from E by EO may be just one of pathways to produce accumulated ecdysteroids.

After moth emergence, the control and RNAi female moths were used to mate with wild male moths, respectively. No significant difference was found in the number of eggs between control and RNAi female moths $(340 \pm 27$ vs. $345 \pm 50, P=0.41)$ (Figure 3D). In addition, we also examined the shell of the eggs using scanning electron microscope (Figure $3 \mathrm{E}$ ). Both two kinds of eggs (oviposited by control and RNAi female adult moths) showed similar shell patterns (Figure 3E). However, the offspring of the RNAi adults showed the significantly decreased hatching rate $(88.37 \pm 6.44 \%$ vs. $70.58 \pm 4.17 \%, P=$ 0.002) (Figure 4A, B). Furthermore, we measured the $20 \mathrm{E}$ concentration of the eggs at $2^{\text {nd }}$ Day and $4^{\text {th }}$ Day after oviposition. Compared with the eggs laid by the control female adults, the offspring of the RNAi adults had significantly lower $20 \mathrm{E}$ level $\left(2^{\text {nd }}\right.$ Day: 0.18 \pm 0.029 vs. $0.11 \pm 0.017 \mu \mathrm{g} / \mathrm{g}$ eggs, $P=0.027 ; 4^{\text {th }}$ Day: $0.63 \pm 0.041$ vs. $0.51 \pm 0.036 \mu \mathrm{g} / \mathrm{g}$ eggs, $P=0.024)$ (Figure 4C). In addition, we found that the development of eggs laid by $E O$ RNAi moths was arrested at organogenesis stage (Figure 4D).

Additionally, the product of EO is 3DE which also can be synthesized into ecdysone. Thus, we injected 3DE to rescue the BmEO RNAi eggs. The newly laid eggs of the BmEO-RNAi adults were 
collected, and then 3DE was injected into the eggs. The result showed that injection with 3DE significantly elevated the hatching rate compared with the control $(52.50 \pm 8.48 \%$ vs. $40.17 \pm 6.02 \%, P=$

A

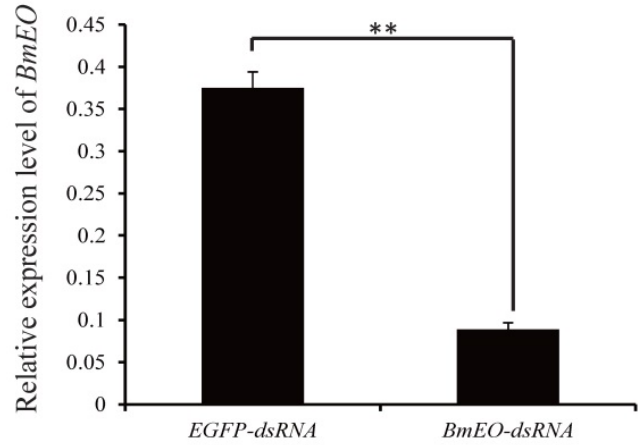

C

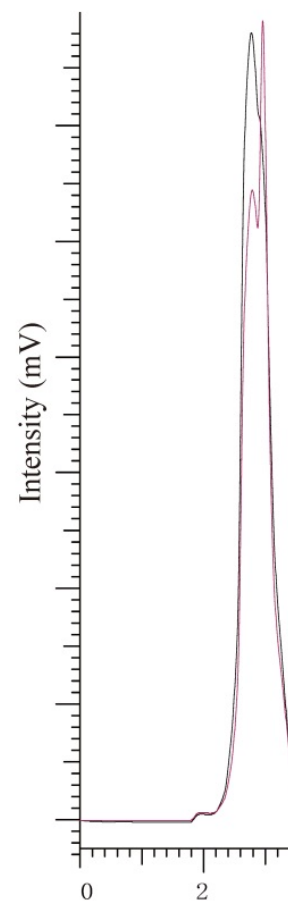

D

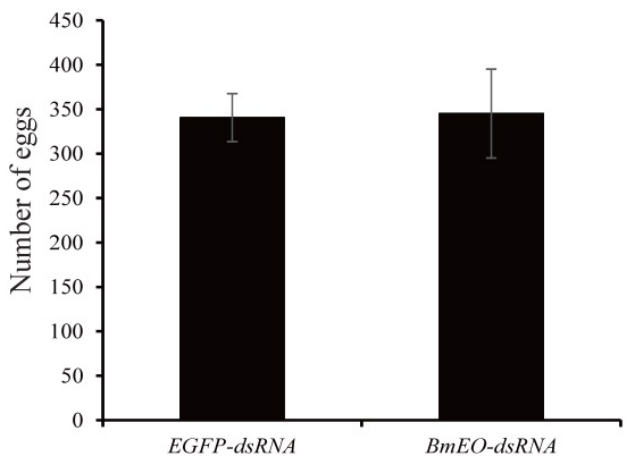

0.015) (Figure 4E). Meanwhile, injection with 3DE could increase the $20 \mathrm{E}$ level at the $2^{\text {nd }}$ Day after treatment $\left(2^{\text {nd }}\right.$ Day: $0.15 \pm 0.038$ vs. $0.24 \pm 0.039 \mu \mathrm{g} / \mathrm{g}$ eggs $P=0.047$ ) (Figure $4 \mathrm{~F}$ ).

B

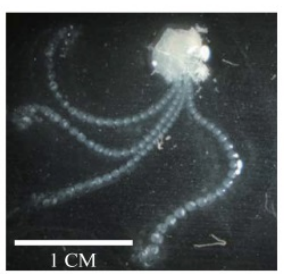

EGFP-dsRNA

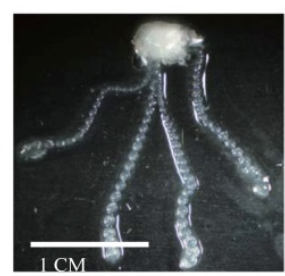

BmEO-dsRNA

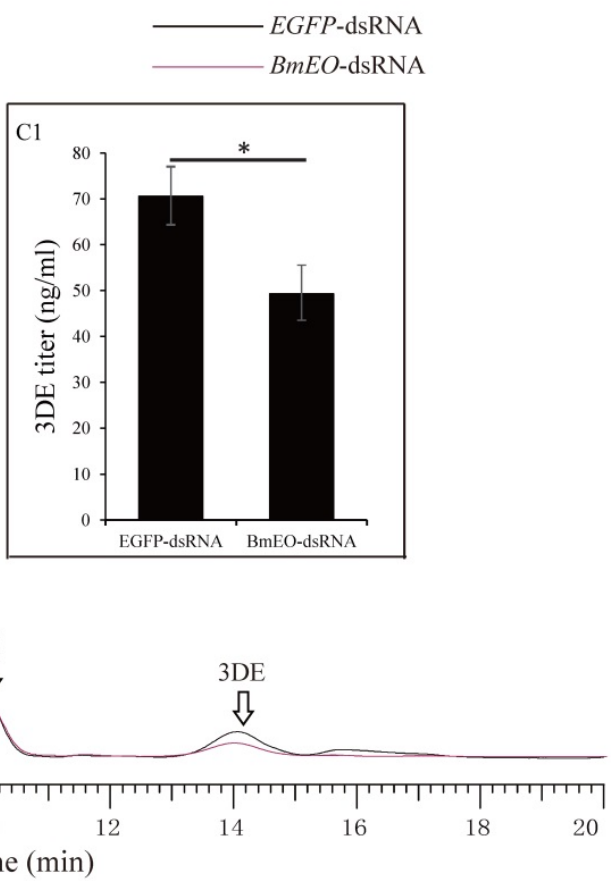

E

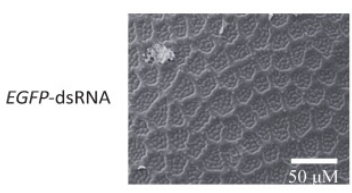

BMEO-dsRNA

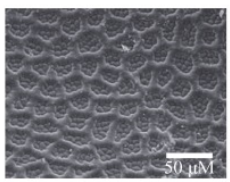

Figure 3. The function of $B m E O$ gene during the female pupal stage. (A) Expression of BmEO gene after RNAi; (B) Phenotype of ovary after RNAi; (C) HPLC analysis of ecdysteroids extracted from eggs of RNAi treated virgin female moth; (D) The number of eggs laid by RNAi female adults; (E) Shell of eggs laid by RNAi adults using a JOEL NeoScope (JCM-5000) scanning electron microscope. For (B), the ovary of the RNAi treated pupae at the fifth day after pupation was dissected; For (C), 3DE, E and 20E were identified by the retention time of their standard sample. We don't have 3D20E standard sample, so we deduced 3D20E peak according to previous studies. UN means unknown products. Inner figure Cl represents the concentration of the 3DE collected from HPLC analysis. 
A

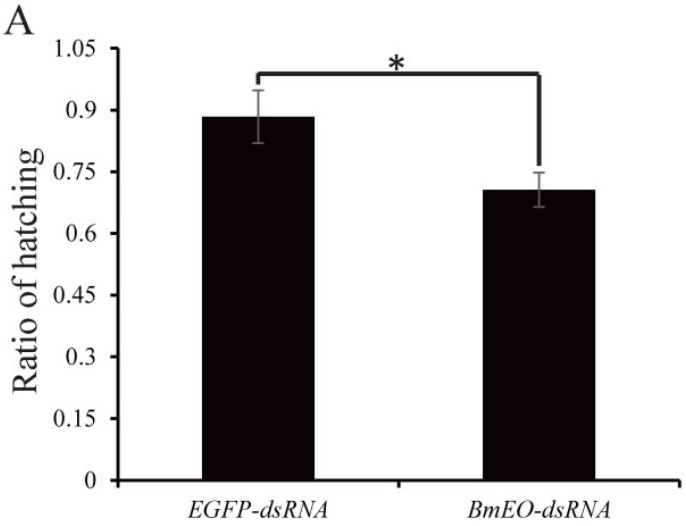

C

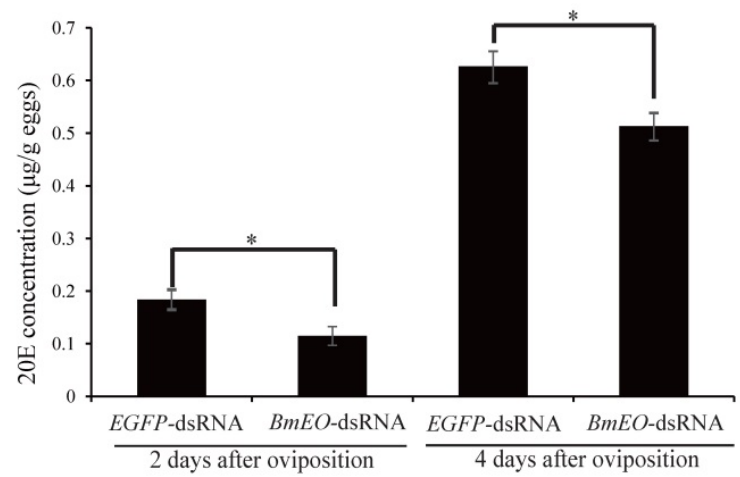

B

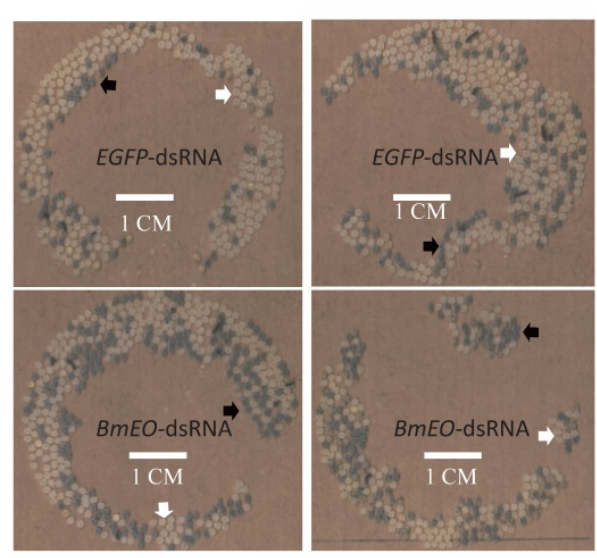

D

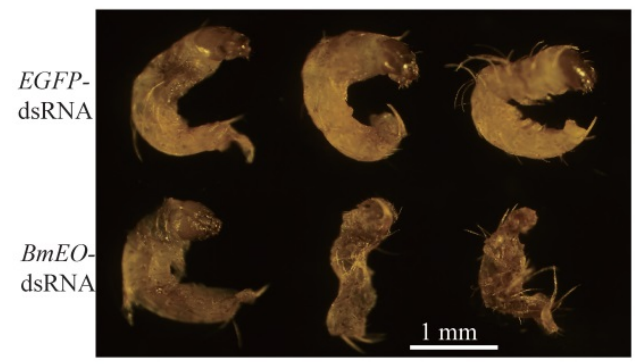

E

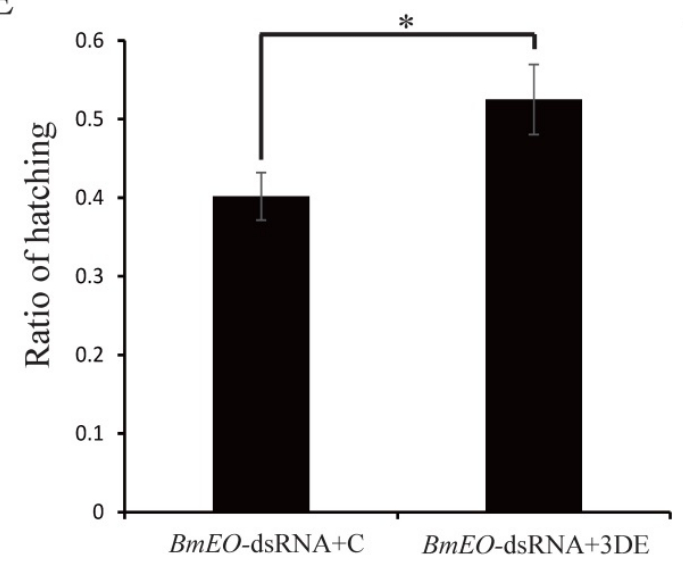

F

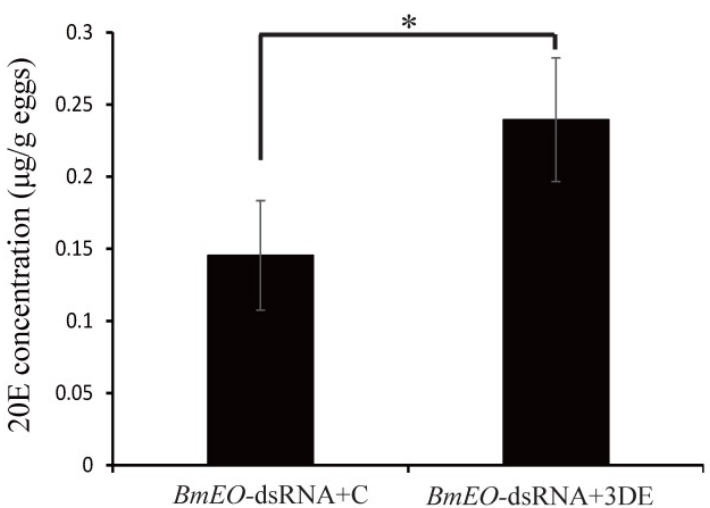

Figure 4. The maternal BmEO-RNAi affects the 20E concentration and hatching rate of offspring. (A) Hatching rate of the eggs laid by RNAi adults; (B) The phenotype of the eggs laid by RNAi adults; (C) $20 \mathrm{E}$ concentration of the eggs laid by RNAi adults; (D) Phenotype of embryos laid by RNAi adults; (E) Hatching rate of BmEO-RNAi adult laid eggs after injection with 3DE; (F) 20E concentration of BmEO-RNAi adults laid eggs after injection with 3DE. For (B), white arrows indicate the hatched eggs, and the black arrows indicate the unhatched eggs; In (D), the embryos at 8-9 days after oviposition were dissected out.

Taken together, our results indicated that maternal BmEO is important for the biosynthesis of $20 \mathrm{E}$ during the embryonic development.

\section{The function of $3 D E-3 \beta$-reductase gene during the embryonic development}

In lepidopteran insects, 3DE can be directly converted into ecdysone by $3 \mathrm{DE}-3 \beta$-reductase. To ascertain the function of the silkworm $3 D E-3 \beta$ reductase gene in the egg, we performed RNAi experiment by injecting the corresponding dsRNAs into the newly laid eggs. Two days after injection, the transcription level of the gene significantly decreased (Figure 5A). Interestingly, similar to the maternal $B m E O$ RNAi experiments, we found that the hatching rate of the eggs injected with $3 D E-3 \beta$-reductase dsRNA was also significantly lower than that of the control $(38.69 \pm 4.66 \%$ vs. $57.35 \pm 6.87 \%, P=0.018)$ (Figure 5B, C). In addition, the $20 \mathrm{E}$ concentration was also examined after injection. The down-regulated expression of the $3 D E-3 \beta$-reductase gene resulted in the significantly declined 20E level compared with the control at embryonic stages ( $2^{\text {nd }}$ Day: $0.35 \pm 0.037$ vs. $0.21 \pm 0.034 \mu \mathrm{g} / \mathrm{g}$ eggs, $P=0.019 ; 4^{\text {th }}$ Day: $0.53 \pm 0.033$ 
vs. $0.40 \pm 0.020 \mu \mathrm{g} / \mathrm{g}$ eggs, $P=0.012$ ) (Figure $5 \mathrm{D}$ ). Furthermore, the decreased $20 \mathrm{E}$ titer significantly affect the normal development of embryos. Development of about $75 \%$ embryos after RNAi treatment was arrested at the stage 20 (segmentation

A

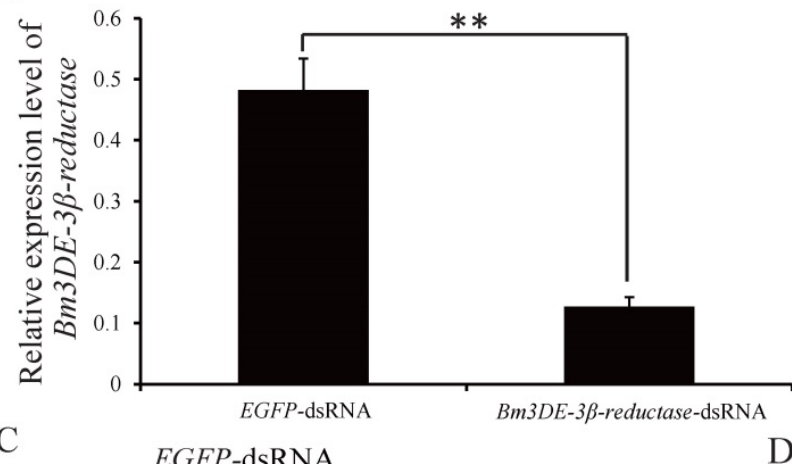

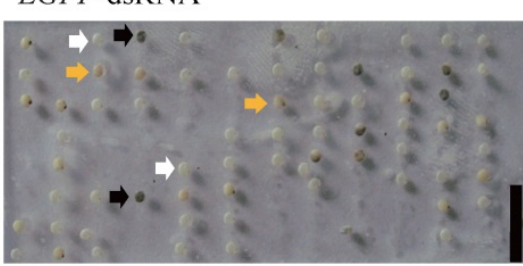

Bm3DE-3 $\beta$-reductase-dsRNA

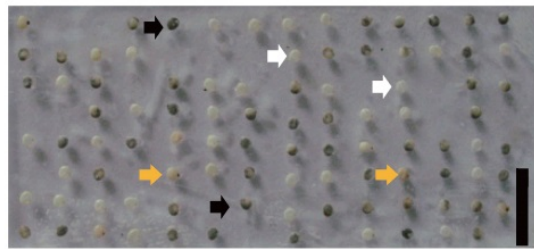

F
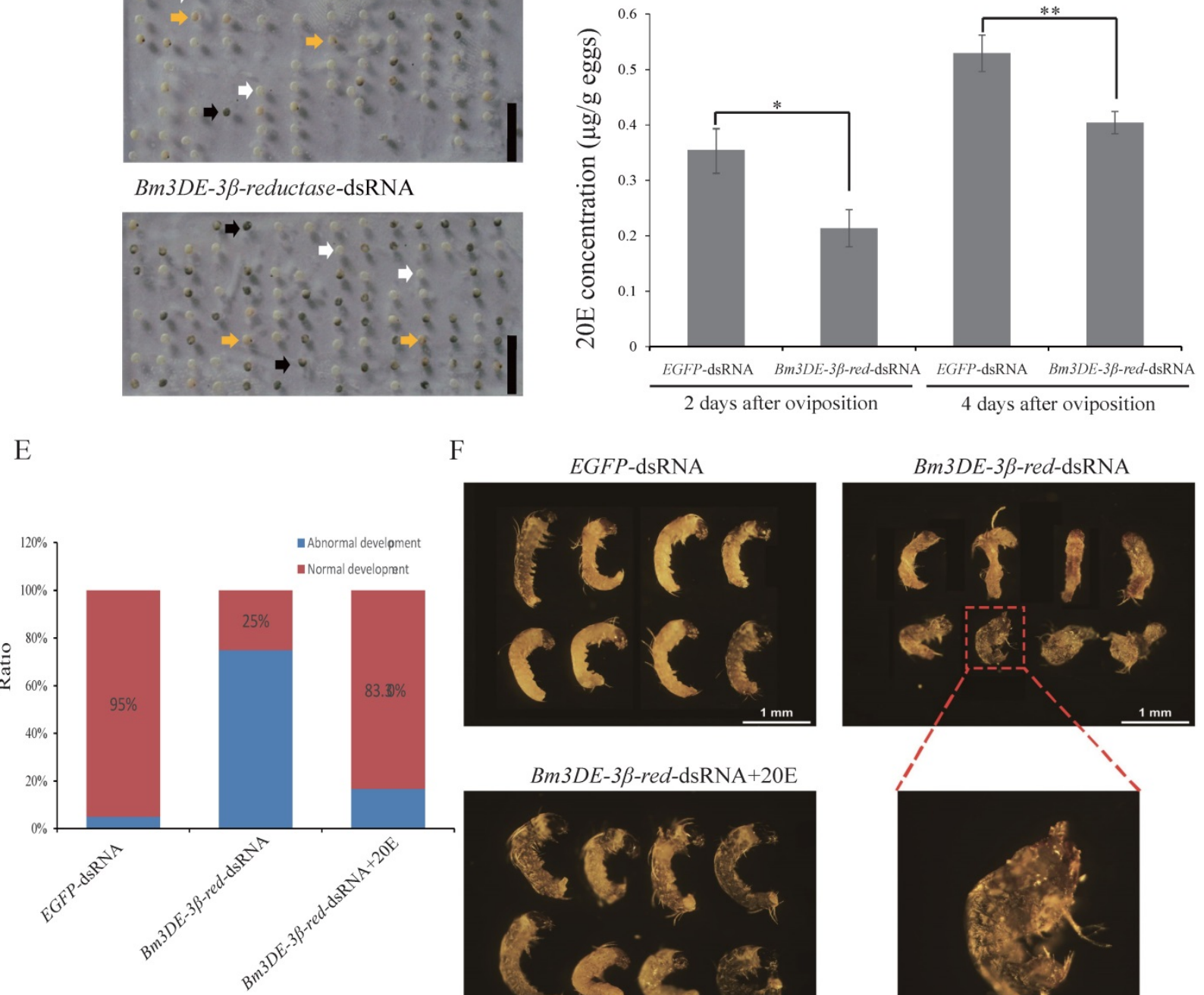

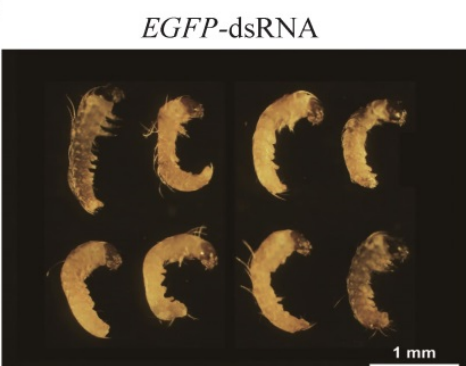

$B m 3 D E-3 \beta$-red-dsRNA $+20 \mathrm{E}$

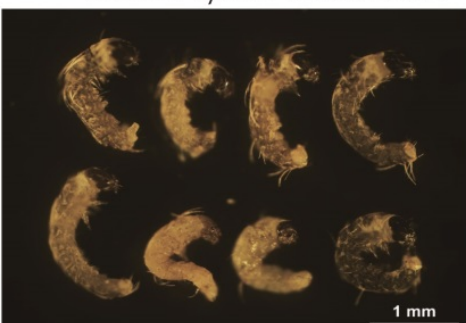

Bm3DE-3 $\beta$-red-dsRNA

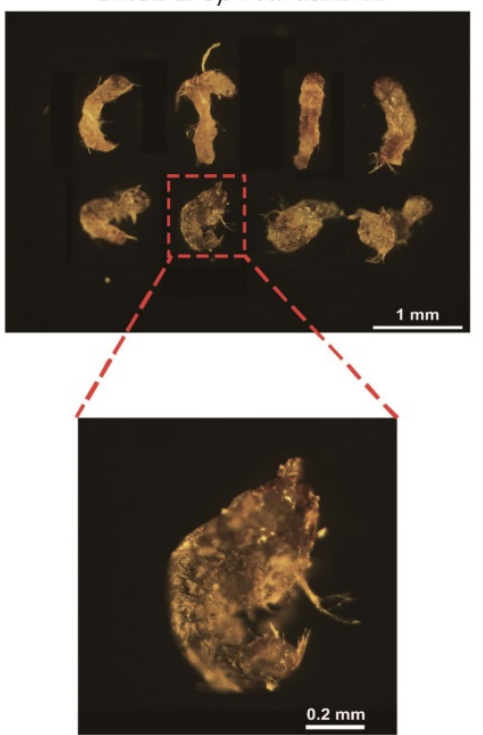

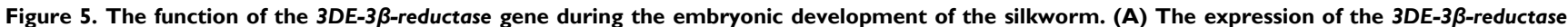

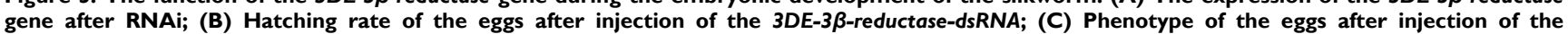
$3 D E-3 \beta$-reductase-dsRNA; (D) 20E concentration of the eggs after injection of the 3DE-3 $\beta$-reductase-dsRNA; (E) Ratio of normal and abnormal development of the embryos after 3DE-3ß-reductase RNAi and rescue experiments; (F) Phenotype of the embryos after RNAi and rescue experiments. For (C), white arrows indicate the hatched eggs, and the black arrows indicate the unhatched eggs and yellow arrows mean the unfertilized eggs. Bar: $1 \mathrm{CM}$. For (F), the embryos at 8-9 days after oviposition were dissected out. 3DE-3ß-reductase-dsRNA+20E means the rescue experiment by co-injected with 3DE-3ß-reductase-dsRNA and enzyme product $(20 \mathrm{E})$. 


\section{Discussion}

In insects, ecdysone is an important hormone for the molting and metamorphosis. Increasing evidence shows that ecdysone is essential for the embryonic development $[9,10]$. However, high concentration of active ecdysone is already present before the formation of PGs during the embryonic development [7]. Thus, ecdysone in the eggs of insects may be maternal origination [21]. Indeed, previous studies revealed that ecdysone-22 phosphate pathway is involved in the synthesis of ecdysone during embryonic development of insects [38, 40].

Similar to the situation in ecdysone-22 phosphate pathway, there is a reversible reaction between ecdysone and 3-dehydroecdysone in ecdysone 3-epimerization pathway, which is catalyzed by EO and 3DE-3 $\beta$-reductase, respectively. We found that $B m E O$ gene was predominantly expressed in the mature ovaries at pupal stage of the silkworm. Previous studies have demonstrated that ecdysone is required for the maturation of ovary in the silkworm [41-43]. High level of 20E titer induces the vitellogenesis but inhibits the choriogenesis [41]. $B m E O$ gene involved in the inactivation of ecdysone was highly expressed in the choriogenic eggs. This indicates that $\mathrm{BmEO}$ may play roles in maintaining the low ecdysone titer during choriogenic stage. However, the down-regulated expression of $B m E O$ gene using RNAi at that stage could not influence the phenotype of the female silkworm, such as ovary development and number of laid eggs. EO could convert ecdysone and 20E into 3DE and 3D20E, respectively. Indeed, we also found that the concentration of $3 \mathrm{DE}$, the intermediate of ecdysone 3-epimerization pathway, decreased after the treatment. 3DE and 3D20E were thought to be the inactive form of ecdysteroids, though several researches showed that 3D20E could induce the expression of ecdysone responsive genes [44, 45]. However, Hiruma et al., (1997) suggested that "the effects of 3D20E can be converted to $20 \mathrm{E}$ by the epidermis, its effects are likely mediated by 20E" [46]. In addition, Baker et al., (2000) found that 3D20E is a relatively poor activator of EcR/USP [47]. Therefore, we thought that female pupa could accumulate inactive 3DE and 3D20E in the eggs.

As shown above, 3DE and 3D20E can also be reversely reduced to ecdysone and $20 \mathrm{E}$ by $3 \mathrm{DE}-3 \beta$-reductase. Indeed, the down-regulated expression of $B m E O$ in maternal pupal ovary lowered the $20 \mathrm{E}$ concentration of its offspring. In addition, rescue experiment by injecting 3DE into the offspring of BmEO RNAi adults could elevate the 20E level and increased the hatching rates. Meanwhile, in the embryo, the down-regulated expression of the
$3 D E-3 \beta$-reductase gene also lowered 20E titer During the silkworm embryonic development, the concentration of $20 \mathrm{E}$ increases at late gastrula stage, and peaks at blastokinesis stage [39]. Previous researches have shown that high titer of ecdysone is essential for germ band retraction, head involution and cuticle deposition [10]. In the domestic silkworm, Kadono-Okuda et al., (1994) also showed that the high titer of accumulated maternal ecdysteroids is essential for embryogenesis and hatching [48]. In this study, the development of embryos with lower $20 \mathrm{E}$ concentration after RNAi treatment arrested at the stage 20 (segmentation of head and thorax) or stage 21 (early blastokinesis) of silkworm embryonic development. Taken together, our results revealed that ecdysone 3-epimerization pathway might have been involved in the synthesis of molting hormone during embryonic development of the silkworm.

Our results showed that ecdysone was converted into $3 \mathrm{DE}$ by $\mathrm{BmEO}$ and stored in the mature ovary. However, it is still unknown which form of 3DE is accumulated in yolk. In insects, ecdysone also can be inactivated by conjugating with glucose, sulphate and phosphate. For example, phosphorylation at the C-22 of ecdysteroids including 20-hydroxyecdysone 22-phosphate (20E22P) and 3-epi-20-hydroxyecdysone 22-phosphate (20E'22P) has been identified in the silkworm [12]. Thus, more studies are needed to confirm whether 3DE has conjugation form in the future.

\section{Supplementary Material}

Supplementary table.

http://www.ijbs.com/v14p1472s1.pdf

\section{Acknowledgments}

We would like to thank Prof. Rene Lafont (Uni-versite Pierre et Marie Curie, Paris, France) for generously providing us the standard sample of the 3DE. This work was supported by the National Natural Science Foundation of China (No. 31272363 to ZZ and No. 31741113 to WS), and funded by Chongqing Research Program of Basic Research and Frontier Technology (No. cstc2017jcyjAX0413).

\section{Author contributions}

WS and ZZ conceived and designed the experiments; CFW and WS performed the experiments; CFW and WS analyzed the data; $\mathrm{ZZ}$ and WS contributed reagents/materials/analysis tools; CFW and WS wrote the paper.

\section{Competing Interests}

The authors have declared that no competing interest exists. 


\section{References}

1. Fukuda S. Hormonal control of molting and pupation in the silkworm. Proceedings of the Imperial Academy. 1940; 8: 417-20.

2. Delbecque J, Lanzrein B, Bordereau C, Imboden H, Hirn M, O'Connor J, et al. Ecdysone and ecdysterone in physogastric termite queens and eggs of Macrotermes bellicosus and Macrotermes subhyalinus. General and Comparative Endocrinology. 1978; 36: 40-7.

3. Imboden H, Lanzrein B, Delbecque JP, Luscher M. Ecdysteroids and juvenile hormone during embryogenesis in the ovoviviparous cockroach Nauphoeta cinerea. General and Comparative Endocrinology. 1978; 36: 628-35.

4. Lagueux M, Hirn M, Hoffmann JA. Ecdysone during ovarian development in Locusta migratoria. Journal of Insect Physiology. 1977; 23: 109-19.

5. Maróy P, Kaufmann G, Dübendorfer A. Embryonic ecdysteroids of Drosophila melanogaster. Journal of Insect Physiology. 1988; 34: 633-37.

6. Ohnishi E, Mizuno T, Chatani F, Ikekawa N, Sakurai S. 2-Deoxy-alpha-ecdysone from ovaries and eggs of the silkworm, Bombyx mori. Science. 1977; 197: 66-7.

7. Ohnishi E, Ohtaki T, Fukuda S. Ecdysone in the eggs of Bombyx silkworm. Proceedings of the Japan Academy. 1971; 47: 413-15.

8. Scalia S, Sbrenna-Micciarelli A, Sbrenna G, Morgan E. Ecdysteroid titres and location in developing eggs of Schistocerca gregaria. Insect Biochemistry. 1987; 17: 227-36.

9. Lagueux M, Hetru C, Goltzene F, Kappler C, Hoffmann J. Ecdysone titre and metabolism in relation to cuticulogenesis in embryos of Locusta migratoria. Journal of Insect Physiology. 1979; 25: 709-23.

10. Kozlova T, Thummel CS. Essential roles for ecdysone signaling during Drosophila mid-embryonic development. Science. 2003; 301: 1911-14.

11. Gharib B, Girardie A, De Reggi M. Ecdysteroids and control of embryonic diapause: changes in ecdysteroid levels and exogenous hormone effects in the eggs of cochineal Lepidosaphes. Experientia. 1981; 37: $1107-08$

12. Makka T, Seino A, Tomita S, Fujiwara H, Sonobe H. A possible role of 20-hydroxyecdysone in embryonic development of the silkworm Bombyx mori. Archives of insect biochemistry and physiology. 2002; 51: 111-20.

13. Tawfik AI, Tanaka Y, Tanaka S. Possible involvement of ecdysteroids in embryonic diapause of Locusta migratoria. Journal of Insect Physiology. 2002; 48: 743-49.

14. Hoffmann JA, Lagueux M. Endocrine aspects of embryonic development in insects. Comprehensive Insect Physiology, Biochemistry and Pharmacology. 1985; 1: 435-60.

15. Rees $\mathrm{H}$. Ecdysteroid biosynthesis and inactivation in relation to function. European Journal of Entomology. 1995; 92: 9-39.

16. Hagedorn $\mathrm{H}, \mathrm{O}^{\prime}$ Connor J, Fuchs M, Sage B, Schlaeger D. The ovary as a source of a-ecdysone in an adult mosquito. Proceedings of the National Academy of Sciences of the United States of America. 1975; 72: 3255-59.

17. Hetru C, Lagueux M, Bang L, Hoffman JA. Adult ovaries of Locusta migratoria contain the sequence of biosynthetic intermediates for ecdysone. Life sciences. 1978; 22: 2141-54.

18. Rubenstein EC, Kelly TJ, Schwartz MB, Woods CW. In vitro synthesis and secretion of ecdysteroids by Drosophila melanogaster ovaries. Journal of Experimental Zoology. 1982; 223: 305-8.

19. Lafont R, Koolman J. Ecdysone metabolism. In: Hoffmann J, Porchet M, eds. Biosynthesis, metabolism and mode of action of invertebrate hormones. Berlin, Heidelberg: Springer; 1984: 196-226.

20. Rees H, Isaac R. Biosynthesis of ovarian ecdysteroid phosphates and their metabolic fate during embryogenesis in Schistocerca gregaria. In: Hoffmann J, Porchet M, eds. Biosynthesis, metabolism and mode of action of invertebrate hormones. Berlin, Heidelberg: Springer; 1984: 181-95.

21. Hanaoka K, Ohnishi E. Changes in ecdysone titre during pupal-adult development in the silkworm, Bombyx mori. Journal of Insect Physiology. 1974; 20: 2375-84.

22. Yamada R, Sonobe H. A novel enzyme ecdysteroid-phosphate phosphatase: purification, kinetic characterization, and molecular cloning. Journal of Biological Chemistry. 2003; 278: 26365-73.

23. Sonobe $\mathrm{H}$, Ohira T, Ieki $\mathrm{K}$, Maeda S, Ito $\mathrm{Y}$, Ajimura M, et al. Purification, kinetic characterization, and molecular cloning of a novel enzyme, ecdysteroid 22-kinase. Journal of Biological Chemistry. 2006; 281: 29513-24.

24. Sakurai S, Warren JT, Gilbert LI. Mediation of ecdysone synthesis in Manduca sexta by a hemolymph enzyme. Archives of Insect Biochemistry and Physiology. 1989; 10: 179-97.

25. Warren JT, Sakurai S, Rountree DB, Gilbert LI, Lee SS, Nakanishi K. Regulation of the ecdysteroid titer of Manduca sexta: reappraisal of the role of the prothoracic glands. Proceedings of the National Academy of Sciences of the United States of America. 1988; 85: 958-62.
26. Takeuchi H, Chen JH, O'Reilly DR, Rees HH, Turner PC. Regulation of ecdysteroid signalling: molecular cloning, characterization and expression of 3-dehydroecdysone 3 alpha-reductase, a novel eukaryotic member of the short-chain dehydrogenases/reductases superfamily from the cotton leafworm, Spodoptera littoralis. Biochemical Journal. 2000; 349: 239-45.

27. Kiriishi S, Rountree D, Sakurai S, Gilbert L. Prothoracic gland synthesis of 3-dehydroecdysone and its hemolymph $3 \beta$-reductase mediated conversion to ecdysone in representative insects. Cellular and Molecular Life Sciences. 1990; 46: 716-21.

28. Milner N, Rees $\mathrm{H}$. Involvement of 3-dehydroecdysone in the 3-epimerization of ecdysone. Biochemical Journal. 1985; 231: 369-74.

29. Machado E, Swevers L, Sdralia N, Medeiros MN, Mello FG, Iatrou K. Prostaglandin signaling and ovarian follicle development in the silkmoth, Bombyx mori. Insect Biochemistry and Molecular Biology. 2007; 37: 876-85.

30. Qiao L, Xiong G, Wang RX, He SZ, Chen J, Tong XL, et al. Mutation of a cuticular protein, BmorCPR2, alters larval body shape and adaptability in silkworm, Bombyx mori. Genetics. 2014; 196: 1103-15.

31. Sun W, Shen YH, Zhou LX, Zhang Z. Ecdysone titer determined by 3DE-3beta-reductase enhances the immune response in the silkworm. Journal of immunology. 2016; 196: 1646-54.

32. Blackford MJ, Dian L. The effects of ingested 20-hydroxyecdysone on the larvae of Aglais urticae, Inachis io, Cynthia cardui (Lepidoptera: Nymphalidae) and Tyria jacobaeae (Lepidoptera: Arctiidae). Journal of Insect Physiology. 1997; 43: 315-27.

33. McKinney DA, Strand MR, Brown MR. Evaluation of ecdysteroid antisera for a competitive enzyme immunoassay and extraction procedures for the measurement of mosquito ecdysteroids. General and Comparative Endocrinology. 2017; 253: 60-9.

34. Takeuchi H, Rigden DJ, Ebrahimi B, Turner PC, Rees HH. Regulation of ecdysteroid signalling during Drosophila development: identification, characterization and modelling of ecdysone oxidase, an enzyme involved in control of ligand concentration. Biochemical Journal. 2005; 389: $637-45$

35. Weirich GF, Feldlaufer MF, Svoboda JA. Ecdysone oxidase and 3-oxoecdysteroid reductases in Manduca sexta: Developmental changes and tissue distribution. Archives of Insect Biochemistry and Physiology. 1993; 23: 199-211.

36. Kiguchi K, Agui N. Ecdysteroid levels and developmental events during larval moulting in the silkworm, Bombyx mori. Journal of Insect Physiology. 1981; 27: 805-7, 9-12.

37. Sonobe $H$, Yamada R. Ecdysteroids during early embryonic development in silkworm Bombyx mori: metabolism and functions. Zoological Science. 2004; 21: 503-16.

38. Yamada $R$, Sonobe $H$. Purification, kinetic characterization, and molecular cloning of a novel enzyme ecdysteroid-phosphate phosphatase. Journal of Biological Chemistry. 2003; 278: 26365-73.

39. Miya K. Early Embryogenesis of embryonic lethal, kidney-shaped egg in Bombyx mori. Zoological Science. 1984; 1: 953.

40. Yamada R, Yamahama Y, Sonobe H. Release of ecdysteroid-phosphates from egg yolk granules and their dephosphorylation during early embryonic development in silkworm, Bombyx mori. Zoological Science. 2005; 22: 187-98.

41. Swevers L, Iatrou K. The ecdysone agonist tebufenozide (RH-5992) blocks the progression into the ecdysteroid-induced regulatory cascade and arrests silkmoth oogenesis at mid-vitellogenesis. Insect Biochemistry and Molecular Biology. 1999; 29: 955-63.

42. Swevers L, Iatrou $\mathrm{K}$. The ecdysone regulatory cascade and ovarian development in lepidopteran insects: insights from the silkmoth paradigm. Insect Biochemistry and Molecular Biology. 2003; 33: 1285-97.

43. Tsuchida K, Nagata M, Suzuki A. Hormonal control of ovarian development in the silkworm, Bombyx mori. Archives of Insect Biochemistry and Physiology. 1987; 5: 167-77.

44. Richards $\mathrm{G}$. The relative biological activities of $\alpha$ - and $\beta$-ecdysone and their 3 dehydro derivatives in the chromosome puffing assay. Journal of Insect Physiology. 1978; 24: 329-35.

45. Spindler KD, Koolman J, Mosora F, Emmerich H. Catalytical oxidation of ecdysteroids to 3-dehydro products and their biological activities. Journal of Insect Physiology. 1977; 23: 441-4.

46. Hiruma K, Bocking D, Lafont R, Riddiford LM. Action of different ecdysteroids on the regulation of mRNAs for the ecdysone receptor, MHR3, dopa decarboxylase, and a larval cuticle protein in the larval epidermis of the tobacco hornworm, Manduca sexta. General and Comparative Endocrinology. 1997; 107: 84-97.

47. Baker KD, Warren JT, Thummel CS, Gilbert LI, Mangelsdorf DJ. Transcriptional activation of the Drosophila ecdysone receptor by insect and plant ecdysteroids. Insect Biochemistry and Molecular Biology. 2000; 30: 1037-43. 
48. Kadono-Okuda K, Amornsak W, Yamashita O. Controlled ecdysteroid accumulation in eggs of the silkworm, Bombyx mori, by an imidazole compound (KK-42), and embryogenesis in these eggs. Archives of Insect Biochemistry and Physiology. 1994; 25: 121-35. 